\title{
On the hygrothermomechanical characterization of polyvinyl acetate
}

\author{
W. G. Knauss and V. H. Kenner \\ Graduate Aeronautical Laboratories, California Institute of Technology, Pasadena, California 91125
}

\begin{abstract}
As a part of a program to understand the mechanisms of failure in time-dependent adhesion and film bonding, the creep compliance of polyvinyl acetate (PVAc) in shear has been determined both as a function of temperature and absorbed moisture. Volumetric expansion as a function of temperature or moisture takeup was also measured. We find that practically realizable changes in moisture content affect both the creep compliance and the swelling of PVAc to a degree comparable to that resulting from realistic changes in temperature. For example, the creep rates (histories) at corresponding times for PVAc subjected to $92 \%$ relative humidity storage are accelerated by approximately four orders of magnitude over those found for the dry material. Moreover, we find within reasonable experimental error that water concentration affects the time scale of creep like temperature through a concentration-dependent shift factor. An attempt is made at discussing the interrelation of temperature- and moisture-induced volume changes.
\end{abstract}

PACS numbers: $62.20 . \mathrm{Hg}$

\section{INTRODUCTION}

In our recent investigations into the fundamental driving mechanims which produce time-dependent failure in adhesive joints and bi-material laminates, the need for a thoroughly characterized model material has arisen. The modeling of material systems requires that this characterization include the following features: (1) the effects of hot (polymeric) film deposition or bond formation with subsequent cooling through the glass-transition temperature $T_{g}$; (2) the effects of operation at elevated and/or cyclic temperatures; and (3) the effects of water, or moisture content.

The first feature will permit assessment of the role which residual stresses incurred during fabrication may play in the failure process. The latter two features translate into an ability to analyze the stresses induced by changes in ambient temperature or moisture level. The history of these stresses depends on viscoelastic material functions which themselves vary with temperature or moisture content. Furthermore, crack-propagation rates in viscoelastic materials depend explicitly on these material functions. ${ }^{1}$ Thus, any analysis of stress history and debond growth requires not only a knowledge of the time-dependent material properties but also the temperature or moisture dependence that these functions exhibit.

We have chosen polyvinyl acetate (PVAc) as a model material. Dilatometrically, this material has a single welldefined glass-transition temperature at approximately $29^{\circ} \mathrm{C}$, and its material properties prove to be suitably temperature and moisture sensitive. Accordingly, we have conducted and report here creep tests in torsion to determine the shear creep compliance $J(t)$ for PVAc both for different levels of temperature and moisture content. The volumetric expansion of PVAc has also been determined both as a function of temperature and absorbed moisture. Finally, we consider the effects which temperature changes and moisture absorption have on the time scale for creep by relating both the moisture and the temperature dependence to the volumetric changes (free-volume change) associated with these variables.

\section{SPECIMEN PREPARATION AND MEASUREMENTS}

\section{A. Specimen preparation}

All PVAc specimens were derived from medium-molecular-weight PVAc, which is obtained from the manufacturer $^{2}$ in the form of clear spherical beads of approximately $0.8 \mathrm{-mm}$ average diameter. These beds were molded at elevated temperature and pressure in a cylindrical cavity $152 \times 15.9 \mathrm{~mm}$ in diameter formed between two steel mold halves. The cylindrical cavity was sealed at one end and fitted with a piston at the other end. After some experimentation, we settled on the molding technique of filling the cavity with PVAc beads at room temperature, heating in a $125^{\circ} \mathrm{C}$ oven for $2 \mathrm{~h}$, inserting the (also heated) piston, and pressurizing the sample via the piston at approximately 68.9 MPa for $5 \mathrm{~min}$ in a hydraulic press. Then the mold was returned to the oven for an additional $2 \mathrm{~h}$, the pressurization was repeated, and the mold was cooled under ambient conditions with piston displacement fixed. This recipe produced uniform cylindrical blanks approximately $102 \mathrm{~mm}$ long, which, while slightly yellowed, exhibited a smooth and glassy fracture surface under microscopic examination at $30 \times$ without revealing the nascent bead structure.

In order to determine the degree to which the molding process produced deterioration of the PVAc, an analysis of molecular weight for both the as-received PVAc beads and the molded blanks was carried out using liquid chromatography. The number average and weight average molecular weights for the beads were found to be 126000 and 353000 , respectively, while corresponding quantities for the molded blanks were 85000 and 268000 , respectively. The decrease in molecular weight during the molding porcess was accompanied by the appearance of ultraviolet (254-nm wavelength) absorbing species of very low molecular weight. Although these observations indicate a definite change of the PVAc during molding, the procedure outlined above produced consistent specimens which proved to have repeatable mechanical properties. Furthermore, the knowledge that no greater changes in molecular weight were encountered un- 
der the relatively harsh molding conditions gave assurance that material changes under the much lower temperature used for subsequent creep measurements would be insignificant.

Torsion creep specimens were machined from the molded cylinders which had a gauge section diameter and length of 7.94 and $51 \mathrm{~mm}$, respectively, and a grip-end diameter of $14.2 \mathrm{~mm}$. Since, as noted above, the glass-transition temperature of $29^{\circ} \mathrm{C}$ for PVAc is only slightly above room temperature, it was found necessary to cool the specimens with compressed air to prevent softening during machining. In a series of preliminary torsion tests, it was found that the machined specimens contained residual stresses which resulted in highly nonuniform test results according to whether the applied torque was in the same or the opposite direction as the torque imposed during the machining (i.e., turning in a lathe). We found that this specimen asymmetry could be removed by annealing the specimen at $40^{\circ} \mathrm{C}$ for $3 \mathrm{~h}$ or more; this was subsequently done with all specimens reported upon.

\section{B. Creep measurements}

All creep testing utilized a creep torsiometer. ${ }^{3}$ This device provides for both the application of either "positive" or "negative" constant torques as low as $6.2 \times 10^{-4} \mathrm{Nm}$ and for the continuous measurement of specimen twist angle. The specimen is housed in a inner chamber which is constructed of 25-mm-thick brass or aluminum walls, floor, and ceiling. This chamber is itself contained in a temperaturecontrolled cabinet so that after thermal equilibrium is established (about $3 \mathrm{~h}$ ) the specimen temperature may be maintained within $\pm \frac{1}{20}{ }^{\circ} \mathrm{C}$ indefinitely. In addition to air vented into the chamber through an air bearing which supports the specimen, a provision is made for injecting additional gas, e.g., dry nitrogen or moist air, into the specimen environment. Thermocouples in the inner chamber measure both dry-bulb and, as necessary, wet bulb temperatures.

Two series of creep tests were conducted on PVAc torsion samples. The first utilized a single specimen which was maintained in the dry state by continuous storage under vacuum except during machining and annealing as described above. Creep test at temperatures ranging from -5 to $+38.6^{\circ} \mathrm{C}$ were carried out over a period of 14 days. Throughout this period the specimen remained in the torsiometer test chamber, which was ventilated continuously with either dry air or dry nitrogen. The creep behavior at $24^{\circ} \mathrm{C}$ was used as a "base-line." This response remained substantially the same for testing at the beginning and at the end of the 14-day period. This practical indication of unchanged specimen properties throughout the test series is consistent with the modest cooling rates used in specimen preparation $\left(10^{\circ} \mathrm{C} / \mathrm{h}\right.$ or less), which should produce a stably equilibrated material. Since the torsiometer allows loading in either of two directions, each new test, after the initial one, was conducted with load applied so as to reduce the cumulative specimen deformation. After each test the specimen was unloaded and allowed to undergo recovery so that the recovery creep superposed on a succeeding test was inconsequential
$(<1 \%)$. This was facilitated by proceeding from the lowest to successively higher temperatures.

The second series of creep tests was conducted using five torsion specimens which were preconditioned at storage relative humidity levels of $32,51,66,81$, and $92 \%$ over suitable saturated salt solutions. Prior to conditioning, each of these specimens was desiccated, annealed, and creep tested at $24^{\circ} \mathrm{C}$ under dry conditions to ensure consistency with the results of the dry-test series. Specimens were weighed in the dry state and intermittently thereafter until the weight stabilized (approximately 10 days). They were then placed in the torsiometer with the test environment maintained at the storage relative humidity level by injecting an appropriate combination of saturated air and dry air into the test chamber at $24^{\circ} \mathrm{C}$. The specimen was weighed immediately after testing to verify that no appreciable weight change had occurred during testing. In the worst instance, the difference between pre-test and post-test weights amounted to $0.049 \%$ of the specimen's dry weight.

\section{Dilatometric measurements}

PVAc strip specimens $70 \times 1.6 \times 12.7 \mathrm{~mm}$ thick, which were formed between polished steel plates in a hot press, were first desiccated and then stored over saturated salt solutions to give both the equilibrium linear dimension increase and the equilibrium mass gain as a function of storage relative humidity. The measurement of dimension changes was made using an optical comparator whose least count was $25 \times 10^{-4} \mathrm{~mm}$, while specimen-weight-change measurement utilized an electronic balance with a least count of 0.1 $\mathrm{mg}$.

The thermal expansion characteristics of PVAc were determined using a mercury dilatometer. ${ }^{4,5}$ In this apparatus, a cylindrical sample of PVAc, $35 \mathrm{~mm}$ long and $6.35 \mathrm{~mm}$

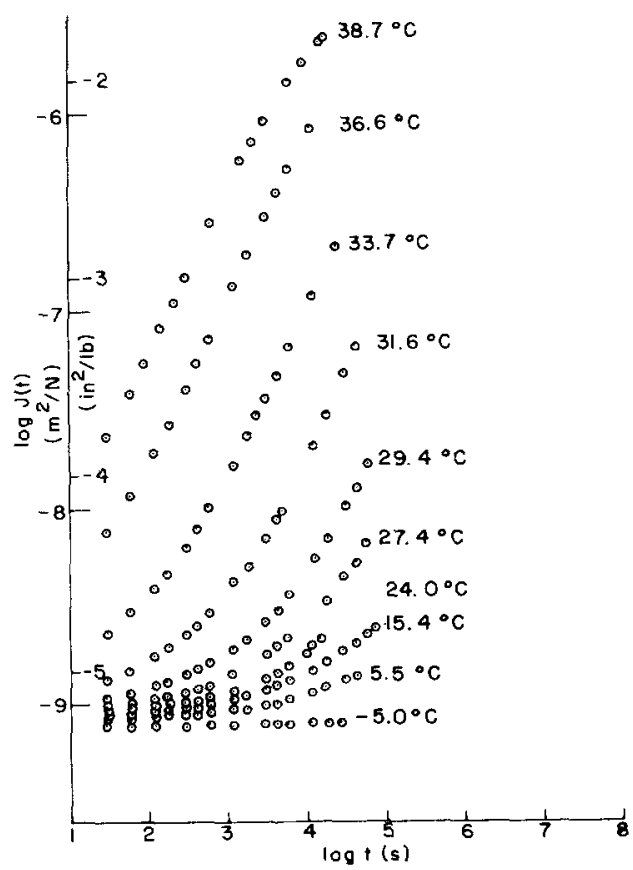

FIG. 1. Creep curves for dry PVAc at several temperatures. 
in diameter, is contained in a small chamber which is otherwise filled with mercury and which is connected to a capillary tube in which the mercury level changes as specimen volume changes due to controlled heating and cooling.

\section{EXPERIMENTAL RESULTS}

Plots of creep compliance $J(t)$ versus time $t$ were derived from the experimental data through the classical relations for torsion of circular cross sections, which give

$$
J(t)=\frac{\gamma(t)}{\tau}=\frac{\pi d^{4} \theta(t)}{32 L T},
$$

where $\gamma(t)$ and $\tau$ are the time-dependent shear strain and constant shear stress, respectively, and $d, \theta(t), L$, and $T$ are the specimen diameter, measured twist angle, specimen gauge length, and applied torque, respectively. Figure 1 presents the creep compliance for dry PVAc with the test temperature as a parameter, while Fig. 2 presents the creep compliance for PVAc at $24^{\circ} \mathrm{C}$ with the moisture weight-gain fraction $\Delta m / m$ as a parameter. The normal error level for these curves is evaluated to be approximately $\pm 2 \%$. Equilibrium swelling and mass gain for PVAc are plotted in Fig. 3 as a function of relative humidity. The thermally induced dilatation for PVAc is exhibited in Fig. 4.

\section{ANALYSIS AND DISCUSSION}

\section{A. Thermomechanical behavior of PVAC}

A master creep curve for PVAc was generated from the data of Fig. 1. First, test temperature was accounted for by shifting each curve vertically by amount $T / T_{0}$, where $T_{0}$ was taken to be $297^{\circ} \mathrm{K}\left(24^{\circ} \mathrm{C}\right)$, according to the notion of the statistical theory of rubber elasticity. Then horizontal shifting was effected relative to the data for $24^{\circ} \mathrm{C}$ to produce the

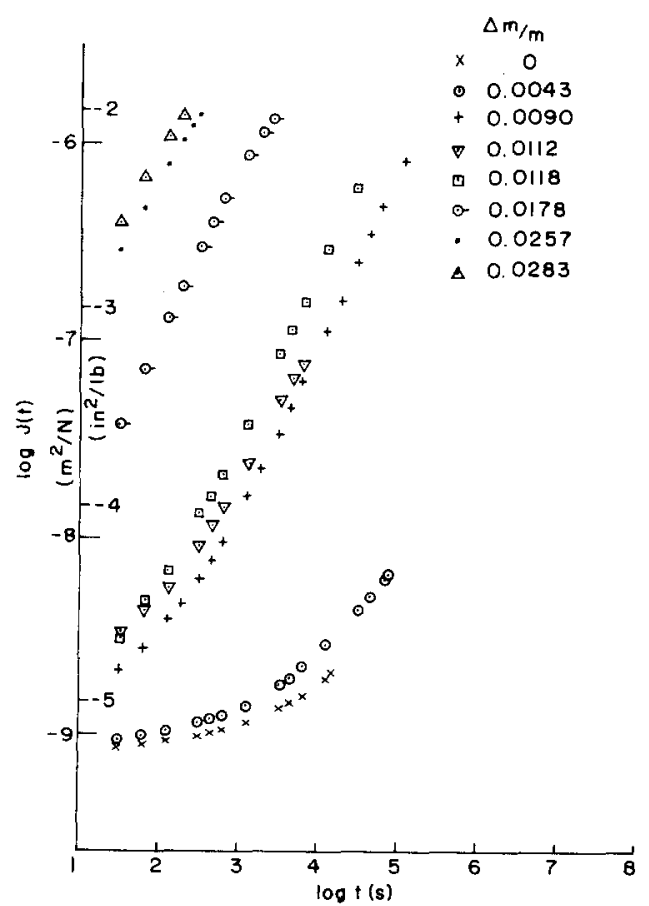

FIG. 2. Creep curves for PVAc at $24^{\circ} \mathrm{C}$ at several absorbed moisture levels.

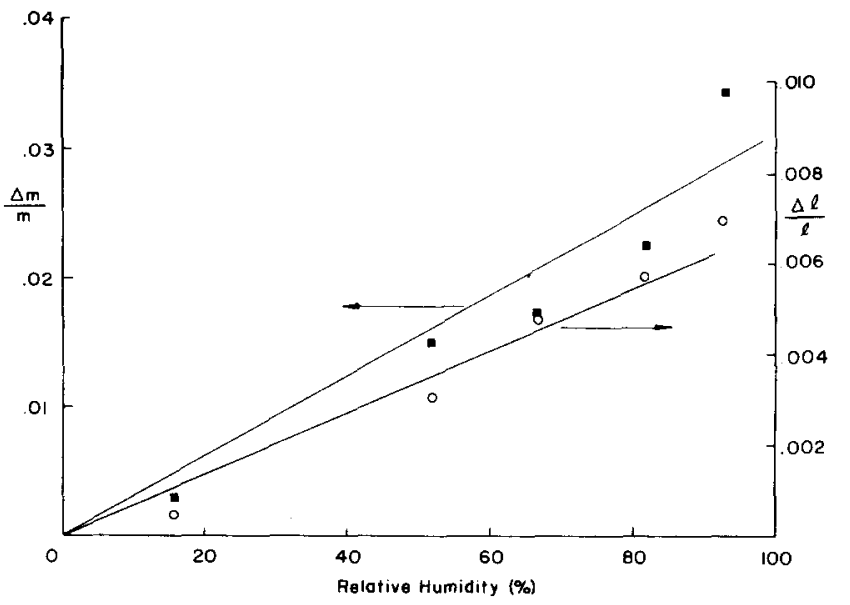

FIG. 3. Moisture takeup and elongation for PVAc as a function of storage relative humidity.

smoothest composite curve from the individual curves; this master curve is presented in Fig. 5.

As Fig. 5 shows, the shifting of creep data to form a master creep curve was accomplished with a good degree of consistency. Thus, we consider PVAc to be reasonably characterized as a thermorheologically simple material so that

$$
J(T, t)=\left(T_{0} / T\right) J\left\{T_{0},\left[t / a_{T}(T)\right]\right\}
$$

Here $a_{T}(T)$ is the temperature-dependent shift factor, which is given in Fig. 6 for dry PVAc. The error bars shown in Fig. 6 represent the shifting uncertainty corresponding to a $\pm 2 \%$ error level for $J(t)$, which is considered representative. The glass-transition temperature of $28.9^{\circ} \mathrm{C}$, determined from the results of the dilatometric measurements presented in Fig. 4, is also shown in Fig. 6. It is seen that at this temperature a change in slope of the shift factor versus temperature data occurs; this slope "discontinuity" has also been observed at the glass transition for a filled epoxy. ${ }^{3}$ Due to the limited data for temperatures above $T_{g}$, it was found that no significant fitting of the WLF $^{6}$ equation was possible. However, the slope of the shift function approaching $T_{\mathrm{g}}$ from $T>T_{g}$ was found to be $-0.45 /{ }^{\circ} \mathrm{C}$, which is equal to $-C_{1} / C_{2}$, the WLF constants $C_{1}$ and $C_{2}$ being referenced to

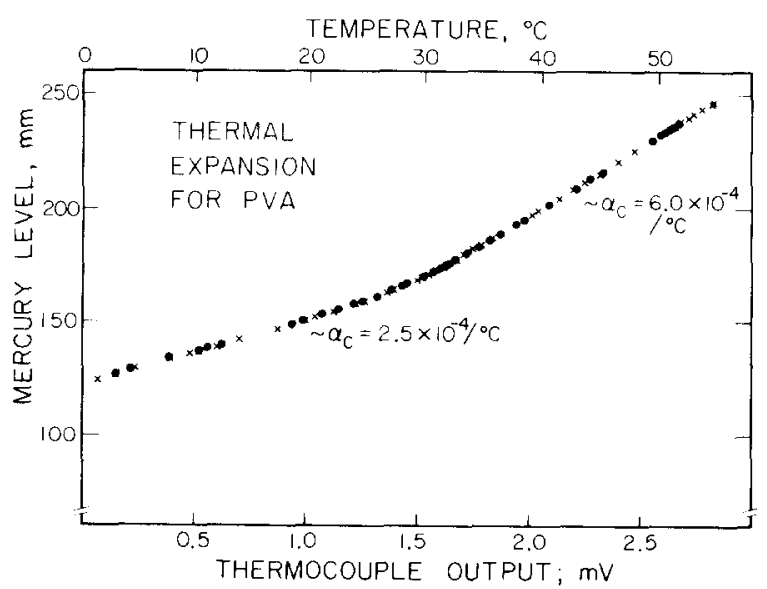

FIG. 4. Thermal-expansion characteristics for PVAc. A change in level of 1 $\mathrm{mm}$ corresponds to a specimen volume change of $13 \times 10^{-5} \mathrm{ml}$. 


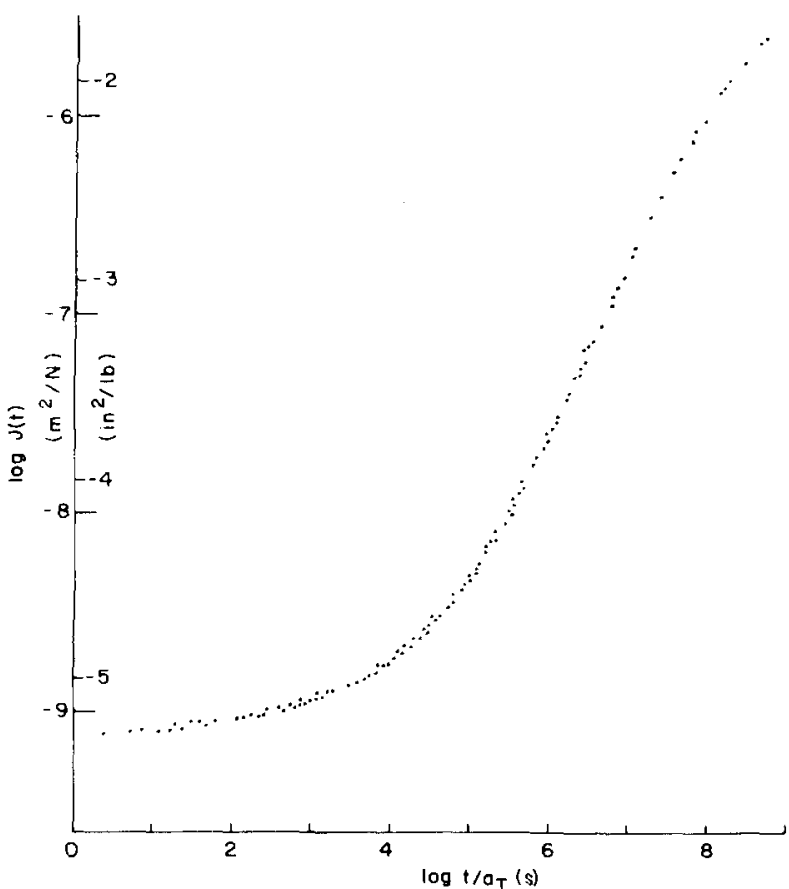

FIG. 5. Master creep curve for dry PVAc at $24^{\circ} \mathrm{C}$, derived from Fig. 1.

the glass-transition temperature. ${ }^{7}$ This value compares to the value of $-0.33 /{ }^{\circ} \mathrm{C}$ calculated from data presented by Ferry. ${ }^{8}$

Figures 5 and 6 may be compared to the experimental results recently presented by Plazek. ${ }^{9}$ These torsional experiments utilized PVAc of molecular weight $6.5 \times 10^{5}$ and were conducted over the temperature range from 37.5 to $154^{\circ} \mathrm{C}$. The recoverable creep compliance curves for both experiments correspond closely in shape, although Plazek's results are shifted approximately $\frac{3}{4}$ decade toward longer time. This difference is qualitatively constants with previous experimental results ${ }^{10}$ and the predictions of entanglement theories ${ }^{11}$ in view of the greater molecular weight of Plazek's PVAc. In addition, we note that our specimen was desiccated but not tested in vacuo; we suspect that a difference in

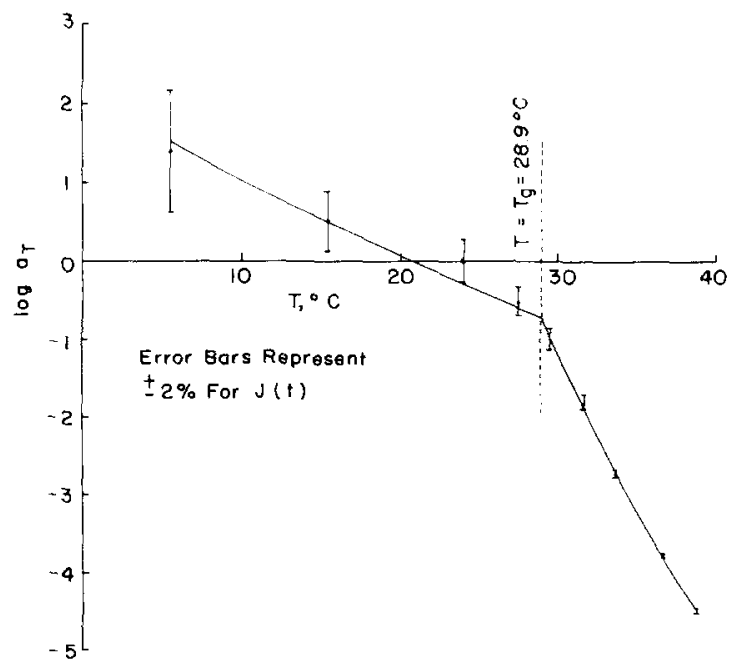

FIG. 6. Temperature shift factor for dry PVAc relative to $24^{\circ} \mathrm{C}$.

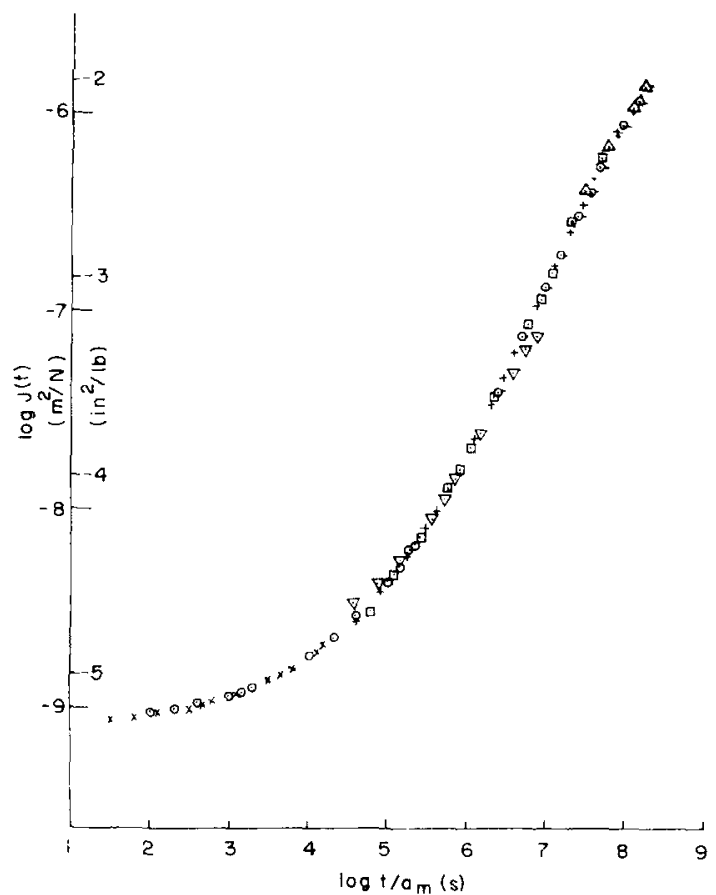

FIG. 7. Master creep curve for dry PVAc at $24^{\circ} \mathrm{C}$ derived from Fig. 2.

"base-line" moisture levels may contribute to the difference. As shown in the sequel, a moisture content change of approximately $\frac{1}{4} \%$ would account for the entire discrepancy. In view of the close temperature control afforded by both our and Plazek's apparatus, we tend to believe that the difference is indeed due to residual moisture which could not be extracted during the period of in vacuo storage.

\section{B. Hygromechanical behavior of PVAC}

The creep curve from Fig. 2 for PVAc at several absorbed moisture levels were shifted horizontally relative to the dry creep curve to produce the master creep curve presented in Fig. 7. With the exception of one slightly irregular run, this procedure resulted in excellent construction of a master curve. The shift factors $a_{m}$ corresponding to Fig. 7 are plotted as a function of the fractional mass gain in Fig. 8 . The error bars shown in Fig. 8 again represent shifting errors corresponding to a $\pm 2 \%$ uncertainty in $J(t)$. There is an

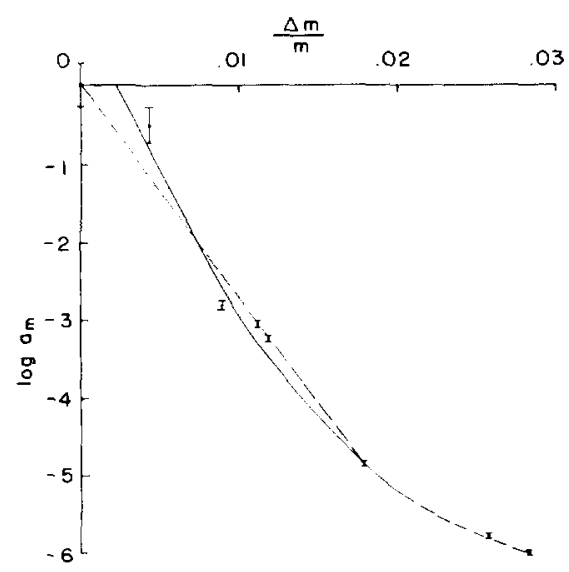

FIG. 8. Moisture absorption shift factor for $24^{\circ} \mathrm{C}$ relative to the dry state. 
inconsistency in the first two data points on this curve, and we show two fits in Fig. 8 according to whether the point at the origin is accepted (dashed curve) or the next point is considered accurate (solid curve). This ambiguity near the origin reflects, first, uncertainty as to the definition of "dry" and, second, increased errors inherent in the shifting procedure for the short-time regime of the master curve.

Horizontal shifting procedures a master curve which represents creep at several moisture absorption levels with dependence on $\Delta m / m$ entirely incorporated in $a_{m}(\Delta m / m)$; therefore, we use the term "hygrorheologically simple material (HSM)" in analogy to the words used in temperaturetime shifting. We thus describe a material for which the moisture dependence is expressible through (in the case of shear creep compliance)

$$
J(\Delta m / m, t)=J_{0}\left\{(\Delta m / m)_{0},\left[t / a_{m}(\Delta m / m)\right]\right\} .
$$

\section{Hygrothermomechanical behavior}

Since both the master curve derived from the dry temperature-varied series of tests (Fig. 5) and that derived from the $24^{\circ} \mathrm{C}$ moisture-varied tests (Fig. 7) are referenced to the dry material at $24^{\circ} \mathrm{C}$, they should, in principle, be identical, i.e., each should predict the extended time creep behavior of dry PVAc at $24^{\circ} \mathrm{C}$. Indeed, when both creep curves are plotted together, as in Fig. 9, one observes that they are essentially identical, the difference between the two being within limits of experimental and plotting errors. Shifting of temperature-and/or moisture-test creep data to produce a single master creep curve has also been acocmplished for other polymers. ${ }^{12,13}$

Since the material response to increased temperature corresponds closely to that resulting from the absorption of moisture, there is a strong indication that the basic mechan-

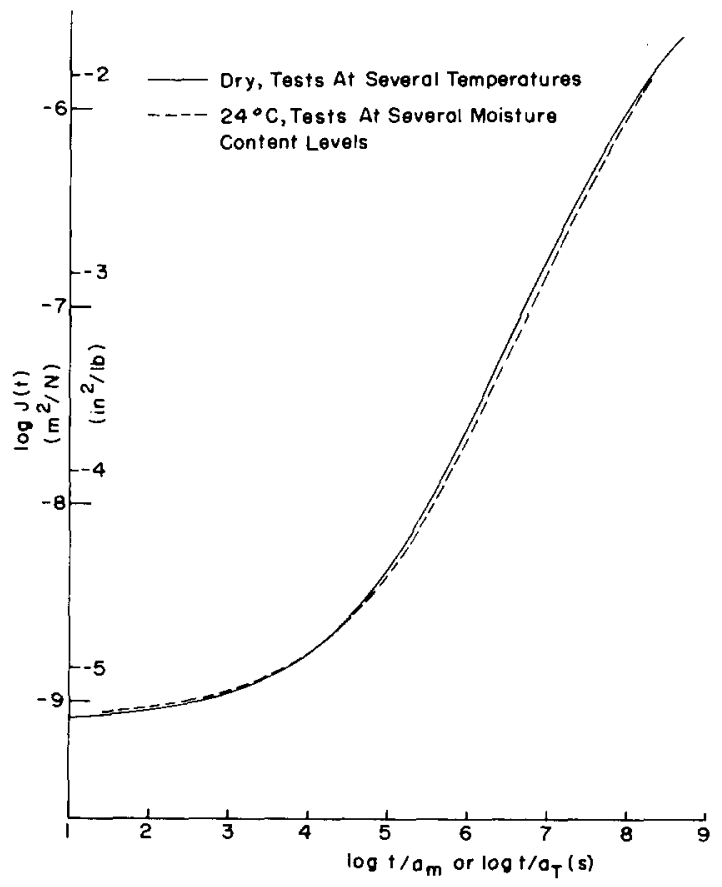

FIG. 9. Comparison of master creep curves for PVAc.

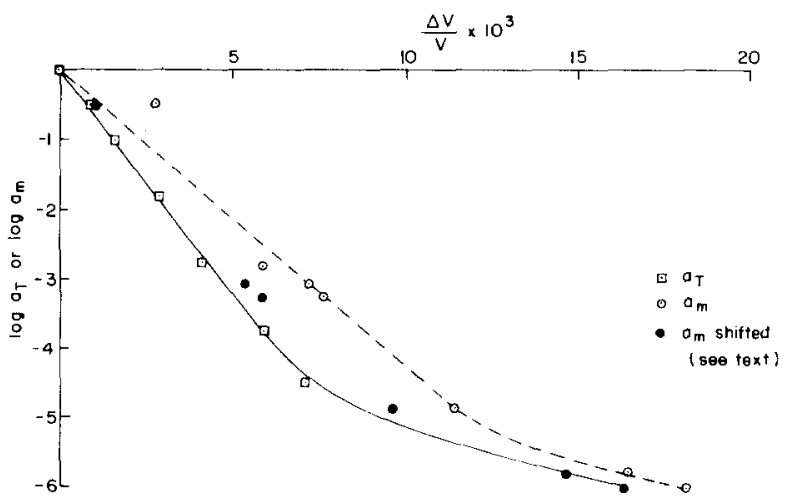

FIG. 10. Shift data from moisture and temperature experiments presented in terms of fractional volume changes.

ics underlying both responses are the same. For example, in view of Fig. 9, it seems unlikely that chemical changes which might occur with moisture absorption have resulted in changed mechanical behavior. Following the relationship between the so-called "free volume" and the time-temperature shift factor $a_{T}{ }^{7}$, we now attempt to establish a commonality between the two sets of creep data on the basis of volume change. Since several representations of free volume have been correlated with experimental observations of various physical phenomena, ${ }^{12.14,15}$ the present comparison is effected simply on the basis of net volume gain relative to the reference state, i.e., relative to dry PVAc at $24^{\circ} \mathrm{C}$.

The volume change due to temperature increase may be calculated from the results of the dilatometric measurements in Fig. 4. The two linear segments of the curve below and above the glass-transition temperature correspond to coefficients of volume expansion of $2.5 \times 10^{-4} / \mathrm{C}$ and $6.0 \times 10^{-4} / \mathrm{C}$, respectively. From these values we readily calculate the fractional volume increase $\Delta v / v$. The data of Fig. 3 may be used to obtain the relation between mass gain due to moisture absorption $\Delta \mathrm{m} / \mathrm{m}$ and the corresponding volume increases $\Delta v / v$. In this case, the straight-line fits shown in Fig. 3 correspond to a ratio

$$
\frac{\Delta v / v}{\Delta m / m}=0.64=\frac{1}{\rho} \frac{\Delta v}{\Delta m},
$$

where $\rho$ is the density of PVAc, which we have determined to be $1.17 \mathrm{~g} / \mathrm{cm}^{3}$. The second equality gives $\Delta v / \Delta m=0.75$ $\mathrm{cm}^{3} / \mathrm{g}$, i.e., the volume increase in the water absorption measurements is equal to three-quarters of the volume of the added water.

Let us now plot the shift factor data of both Figs. 6 and 8 in terms of the fractional volume increase $\Delta v / v$ relative to dry PVAc at $24^{\circ} \mathrm{C}$ in Fig. 10. In the case of $a_{m}$, the degree of linearity of the $\log a_{m}-v s-\Delta v / v$ curve simply corresponds to that exhibited by the $\log a_{m}$-vs- $\Delta m / m$ data presented in Fig. 8. However, the linearity exhibited by $\log a_{T}$ in Fig. 10 represents a "straightening" from the antecedent data of Fig. 6. Thus, we emphasize that while shift data in the trans- $T_{g}$ range exhibit a discontinuity of slope at $T_{g}$, these data are continuous when recast in terms of volume change.

Disregarding, for the moment, the possibility that the "dry" specimen was not entirely free of water, ${ }^{16}$ one obtains 
the relation shown dotted in Fig. 10 for the shift function $a_{m}$. The shift function derived from temperature variations is plotted by squares. By comparing these two relations one would be led to confirm that volume dilatation by water leads to less "free volume" for molecular motion than dilatation through temperature would provide.

Alternately, if our so-called "dry" material was not truly free of water in spite of prolonged desiccation, then a correction of water content by an initial small amount of about $0.27 \%{ }^{17}$ would bring the temperature and moisture shift data into good agreement (solid circles and square, Fig. 10).

Clearly, the necessity of further work on preparation of water-free specimens is indicated to completely resolve this uncertainty. However, it emerges fairly clearly already that changes in rheological behavior resulting from changes in temperature and moisture are coupled through the free volume of the material.

The present PVAc is similar to the nylon reported on by Williams and Bender ${ }^{12}$ in the sense that $\Delta v / \Delta m$ is initially equal to $0.75 \mathrm{~cm}^{3} / \mathrm{g}$ for water absorption in both materials. If the view that free volume consists of voids is taken, ${ }^{12}$ then the free volume decreases as absorbed water fills voids. The presence of water in these voids makes either no or only slight difference according to the current results. Thus, for purposes of determining changed creep compliance, we consider all volume increases due to absorption to represent "effective free volume." This seems consistent with a notion of ease of movement of large molecules being dependent on space between them, with relatively very small water molecules providing little, if any, change from void insofar as the present quasistatic and equivoluminal motion is concerned.

Consider now the usual representation ${ }^{7}$ of the shift factor as a function of free volume fraction $f$ through

$$
\log a=c\left(1 / f-1 / f_{0}\right),
$$

where $c$ is constant and $f_{0}$ represents the fraction of free volume at reference conditions. If, in view of the above discussion, we suppose that volume change governs shifting regardless of its origin, then the fractional free volume $f$ would, to first order, change additively due to temperature and moisture. Thus, if $f_{T}$ and $f_{m}$ represent the free-volume frac- tion due to temperature and moisture, respectively, then $f=f_{T}+f_{m}$. One concludes then from Eq. (5) that a combined shift factor $a$ is not merely a product of the separate shift factors $a_{T}$ and $a_{m}$, i.e., that shifting (on a log-log plot) will not be simply the sum of moisture and temperatures shifts. This is consistent with the empirical representation of the temperature- and moisture-dependent shift factor observed by Maksimov et al. ${ }^{13}$ for polyester resin.

\section{ACKNOWLEDGMENTS}

The authors wish to express their gratitude to the Air Force Office of Scientific Research (Lt. Col. J. Morgan), and the Jet Propulsion Laboratory (Dr. J. Moacanin) and the Department of Energy, who supported this work through Contracts F49620-77-C-0051 and 49-767-1D002-0-3460, respectively. We also gratefully acknowledge the assistance of M. Sarbolouki of JPL for performing the liquid chromatography and I. Emri and L. Heymans of Caltech for conducting the measurements leading to Figs. 3 and 4, respectively.

'W. G. Knauss, in Deformation and Fracture of High Polymers, edited by H. H. Rausch, J. A. Hassell, and R. I. Jaffee (Plenum, New York, 1974), p. 501; in particular comment 4, p. 538.

${ }^{2}$ Polysciences, Inc., Warrington, Pa.

${ }^{3}$ V. H. Kenner and W. G. Knauss (unpublished).

${ }^{4}$ W. G. Knauss, V. H. Kenner, and L. J. Heymans, GALCIT SM 78-12

(California Institute of Technology) (unpublished).

'N. Bekkedahl, J, Res. Nat. Bur. Stand. 42, 145 (1949)

'M. L. Williams, R. F. Landel, and J. D. Ferry, J. Am. Chem. Soc. 77, 3701 (1955).

'J. D. Ferry, Viscoelastic Properties of Polymers (Wiley, New York, 1961).

${ }^{8}$ See Ferry's (Ref. 7) discussion, p. 218

${ }^{9}$ D. J. Plazek, Soc. Rheol. 1979 Fall Meeting.

${ }^{10} \mathrm{~K}$ Ninomiya, J. Colloid Sci. 14, 49 (1959).

"'S. D. Hong, J. Appl. Phys. 48, 4019 (1977).

${ }^{17}$ M. L. Williams and M. F. Bender, J. Appl. Phys. 36, 3044 (1965).

${ }^{13}$ R. D. Maksimov, E. A. Sokolov, and V. P. Mochalor, Polym. Mech. 11, 334 (1976).

${ }^{14}$ F. N. Kelley and F. Bueche, J. Polym. Sci. 50, 549 (1961)

${ }^{15} \mathrm{~W}$. Brostow, Research Conference in Chemical Engineering at California Institute of Technology, 1980 (unpublished).

${ }^{16}$ See Sec. IV A.

${ }^{17}$ Corresponding to a volume change of $0.18 \%$. 